\title{
Strong Stability Analysis of Linear Delay Difference Equations with Multiple Time Delays
}

\author{
Yunxia Song, Wim Michiels, Bin Zhou, Senior Member, IEEE, Guang-Ren Duan, Fellow, IEEE
}

\begin{abstract}
This paper is concerned with the strong stability problem of linear continuous-time delay-difference equations with multiple timedelays. A family of linear matrix inequalities (LMIs), indexed by a positive integer $k$, is derived to assess strong stability. A time-domain interpretation of the proposed LMI based condition is given in terms of a quadratic integral Lyapunov functional, which allows to reveal relations with an existing result. The LMI condition can easily be reformulated in a form where the dependence on the coefficients of the delay-difference equation is affine, which is instrumental to establishing a sufficient LMIcondition for robust strong stability of delay-difference equations with norm bounded uncertainty. A necessary and sufficient condition for robust strong stability is also given, in the form of a structured singular value characterization. Examples are given to illustrate the effectiveness of the presented results.
\end{abstract}

Index Terms-Strong stability, Robust strong stability, Delay-difference equations, Quadratic integral Lyapunov functional.

\section{INTRODUCTION}

W e consider continuous-time linear delay-difference equations with discrete delays of the form

$$
x(t)=\sum_{i=1}^{N} A_{i} x\left(t-r_{i}\right), \quad t \geq 0,
$$

where $r_{i}>0$ are constants and $A_{i}$ are $n \times n$ matrices for $i=1,2, \ldots, N$. Delay-difference equations often appear in coupled differential-difference equations [20], [28], neutral time-delay systems [17], and integral equations or difference equations with distributed delays [3]. Applications can be found in lossless propagation along electrical lines [8] and water pipes [39], and in other models for physical phenomena involving conservation laws [9], [13]. Delay-difference equations play a significant role in the stability theory of time-delay systems. In particular, a necessary condition for the stability of coupled differential-difference equations [36], [37] and delay differential equations of neutral type [19], [30], [34], [42] is given by the stability of the associated delay difference equation. Therefore, the study of delay-difference equations has attracted considerable attention in recent years (see e.g. [11], [40].

The stability analysis of delay-difference equations deserves special care, as exponential stability may be sensitive to infinitesimal delay perturbations. This sensitivity problem led to the introduction of the notion of strong stability. More precisely, the zero solution of (1) is strongly (exponentially) stable if there exists a real number $\varepsilon>0$ such that the zero solution of system

$$
x(t)=\sum_{i=1}^{N} A_{i} x\left(t-\left(r_{i}+\delta r_{i}\right)\right)
$$

is exponentially stable for all $\left(\delta r_{1}, \ldots, \delta r_{N}\right) \in \mathbf{R}^{N}$ satisfying $\left|\delta r_{i}\right| \leq \varepsilon$ for $i=1, \ldots, N$ [23]. A well-known spectral condition

Y. X. Song, B. Zhou (Corresponding author) and G. R. Duan are with the Center for Control Theory and Guidance Technology, Harbin Institute of Technology, Harbin 150001, China (e-mail: binzhoulee@163.com, binzhou@hit.edu.cn).

W. Michiels is with the Department of Computer Science, K. U. Leuven, B-3001 Heverlee, Belgium (e-mail: wim.michiels@cs.kuleuven.be).

This work was supported in part by the National Natural Science Foundation of China under grant number 61773140. has been established in the literature [2], [24], which expresses that (1) is strongly stable if and only if

$$
\sup _{\left(\theta_{1}, \theta_{2}, \ldots, \theta_{N}\right) \in[0,2 \pi]^{N}} \rho\left(\sum_{i=1}^{N} A_{i} \mathrm{e}^{\mathrm{j} \theta_{i}}\right)<1,
$$

where $\rho(A)$ denotes the spectral radius of matrix $A$ and $\mathrm{j}$ is the imaginary unit. Recently, several contributions have been made to this topic. In [35] expression (2) was extended to the case where the delay parameters in (1) might be linear combinations of a possibly smaller number of independent delays. In [25] a strong stability criterion was established by deciding positive definiteness of a trigonometric matrix polynomial, which is translated into a converging hierarchy of LMIs. The conditions in [25] rely on computing the coefficients of the characteristic function and are thus not explicitly expressed as a function of the coefficient matrices, which makes their use for robust stability analysis more difficult. The stability of (1) has also been studied via Lyapunov-Krasovskii functionals (LKF), leading to sufficient conditions in terms of LMIs, see [12], [14], [16], [29], [32] and [33]. In [27] an internally positive representation approach was introduced to such a system, resulting in a delay-independent sufficient condition. The robust strong stability problem of delaydifference equations with uncertainties on the coefficient matrices has also been addressed in the literature. For example, an optimization based procedure for assessing robust strong stability was presented in [1], as a by-product of computing strong $\mathcal{H}_{\infty}$ norms used for robust performance analysis.

We note that expression (2) can also be interpreted as a robust stability criterion for discrete-time linear system $x(k+1)=$ $\left(A_{0}+z_{1} A_{1}+\cdots+z_{N} A_{N}\right) x(k)$, with $\left|z_{i}\right| \leq 1, i=1,2, \ldots, N$ considered as uncertainties [25]. The corresponding problem in the continuous-time setting was studied in [4], and nonconservative LMI-based conditions were established by a parameter-dependent Lyapunov approach. This approach also has potential for discretetime linear systems, which was remarked in [10], but, to the best of our knowledge, it has not been explicitly worked out in the literature. Recently, a necessary and sufficient LMI condition for strong stability of delay-difference equation (1), inferred from the robustness analysis of linear systems with scalar uncertainties, was established in [41] for the special case of two delays, along with a sufficient condition for robust strong stability. Other methods developed in the literature, for example, parameter dependent polynomial approach [6], [21], sums of squares techniques [22], have also been used for robust stability of uncertain linear time-invariant systems.

Motivated by the aforementioned discussions, the present paper is devoted to the strong stability and robust strong stability problem of linear continuous-time delay-difference equations with multiple timedelays. The main contributions are as follows. First, a family of linear matrix inequalities, is presented for strong stability of (1), which, by virtue of the Yakubovich-Kalman-Popov (YKP) lemma, can be expressed in a form with affine dependence on the coefficients of the delay-difference equation. Second, a time-domain interpretation of the proposed LMI condition is given in terms of a quadratic integral Lyapunov functional, establishing a relation between the LMI 
condition and a result in [12]. Third, a sufficient LMI condition is presented, guaranteeing robust strong stability when the matrices in (1) are subjected to bounded uncertainties. Finally, motivated by the fact that the robust strong stability of a system can be measured by the distance to instability (stability radius) [7], [26], we derive a necessary and sufficient condition for robust strong stability of delay-difference equations in terms of the real structured singular value [31].

The remainder of this paper is organized as follows: Section II presents a family of LMIs and a time-domain interpretation of the proposed LMI condition. The robust strong stability problem with norm bounded uncertainties is discussed in Section III. Examples are presented in Section IV to illustrate the obtained results. Section V concludes the paper.

Notation: Matrices $I_{n}, 0_{n}, 0_{n \times p}$ are the $n \times n$ identity matrix, the $n \times n$ zero matrix, and the $n \times p$ zero matrix, respectively. The symbol $\otimes$ denotes the Kronecker product. The power of the Kronecker product is defined recursively as $M^{0 \otimes}=1, M^{p \otimes} \triangleq M^{(p-1) \otimes} \otimes M$. The transpose and conjugate-transpose of $P$ are denoted by $P^{\mathrm{T}}$ and $P^{\mathrm{H}}$ respectively. We denote by $\mathbf{Z}, \mathbf{Z}^{+}, \mathbf{C}$ and $\mathbf{R}$ sets of nonnegative integers, positive integers, complex and real numbers, respectively. The standard notation $P>0(\geq 0)$ means that $P$ is a symmetric positive definite (semi-definite) matrix. The symbol $|a|$ refers to the modulus of complex number $a$. The set of symmetric real matrices of size $n \times n$ is denoted by $\mathbf{S}^{n}$. The notation $\operatorname{diag}\left\{P_{k}\right\}, k=1, \ldots, N$ stands for a block-diagonal matrix with diagonal blocks $P_{k}$. For any $k \in \mathbf{Z}^{+}$, let $L_{k}, R_{k} \in \mathbf{R}^{k \times(k+1)}$ be defined by

$$
L_{k}=\left[\begin{array}{ll}
I_{k} & 0_{k \times 1}
\end{array}\right], R_{k}=\left[\begin{array}{ll}
0_{k \times 1} & I_{k}
\end{array}\right] .
$$

For any $u \in \mathbf{C}, u^{[k]}$ is defined as

$$
u^{[k]} \triangleq\left[\begin{array}{llll}
1 & u & \cdots & u^{k-1}
\end{array}\right]^{\mathrm{T}} .
$$

\section{Strong Stability Condition}

We denote by $x(t, \varphi)$ the unique piecewise right-continuous solution with piecewise right-continuous, bounded initial condition $\varphi$ $\in \mathcal{C}\left\{[-r, 0), \mathbf{R}^{n}\right\}, r=\max \left\{r_{i}\right\}$. By combining an extension of the powerful methodology of [4] to the discrete-time setting with the interpretation of (2) as a robust stability criterion for a discretetime LTI system, we establish a novel LMI-based condition for the strong stability of delay-difference equation (1) which, as we shall see in the next section, naturally extends towards problems with uncertainty on the coefficient matrices. Moreover, we give a timedomain interpretation of the proposed LMI condition and establish connections with an existing result.

\section{A. Strong Stability Condition in terms of LMIs}

We first recall a definition from [4].

Definition 1: Let $\tilde{z}_{k}=z_{N-1}^{[k]} \otimes z_{N-2}^{[k]} \otimes \cdots \otimes z_{1}^{[k]} \otimes I_{n}$. The function $x^{\mathrm{T}}(t) P(z) x(t)$ with

$$
P(z) \triangleq \tilde{z}_{k}^{\mathrm{H}} P_{k} \tilde{z}_{k},
$$

where $x(t) \in \mathbf{R}^{n}, z=\left(z_{1}, z_{2}, \ldots, z_{N-1}\right) \in \mathbf{C}^{N-1}$ and $P_{k} \in \mathbf{S}^{k^{N-1} n}$, is called a polynomial parameter-dependent quadratic (PPDQ) function. The integer $k-1$ is called the degree of the PPDQ function of $P(z)$.

We can rewrite (2) in the form

$$
\sup _{\left(\theta_{1}, \theta_{2}, \ldots, \theta_{N}\right) \in[0,2 \pi]^{N}} \rho(A(z))<1,
$$

where $A(z)=A_{1}+A_{2} z_{1}+\cdots+A_{N} z_{N-1}$ with $z=$ $\left(z_{1}, z_{2}, \ldots, z_{N-1}\right) \in \mathbf{C}^{N-1},\left|z_{i}\right|=1, i=1, \ldots, N-1$. Then it follows from (2) that (1) is strongly stable if and only if

$$
\rho(A(z))<1
$$

where $z=\left(z_{1}, z_{2}, \ldots, z_{N-1}\right)$. Based on (6) and the concept of PPDQ function in Definition 1, we will show that the strong stability of (1) is equivalent to the existence of a PPDQ function $x^{\mathrm{T}}(t) P(z) x(t)$ such that

$$
A^{\mathrm{H}}(z) P(z) A(z)-P(z)<0 .
$$

Moreover, as shown in [4], matrix inequality (7) can be transformed into a set of parameter-independent linear matrix inequalities by applying the discrete-time KYP lemma [38].

For $l=1,2, \ldots, N-1$, we denote

$$
\begin{aligned}
\mathscr{B}_{k} & =L_{k}^{(N-1) \otimes} \otimes I_{n}, \\
\mathscr{C}_{k} & =L_{k}^{(N-l) \otimes} \otimes I_{(k+1)^{l-1} n}, \\
\mathscr{D}_{k} & =L_{k}^{(N-1-l) \otimes} \otimes R_{k} \otimes I_{(k+1)^{l-1} n},
\end{aligned}
$$

which are obviously independent of $\left(A_{1}, A_{2}, \ldots, A_{N}\right)$ and

$$
\begin{aligned}
& \mathscr{A}_{k}\left(A_{1}, \ldots, A_{N}\right) \\
& =L_{k}^{(N-1) \otimes} \otimes A_{1}+\sum_{i=2}^{N}\left(L_{k}^{(N-i) \otimes} \otimes R_{k} \otimes L_{k}^{(i-2) \otimes} \otimes A_{i}\right),
\end{aligned}
$$

which is a linear function of $\left(A_{1}, A_{2}, \ldots, A_{N}\right)$. Denote $\mathscr{A}_{k}=$ $\mathscr{A}_{k}\left(A_{1}, A_{2}, \ldots, A_{N}\right)$ for notation simplicity. For $N$ symmetric matrices $P_{k}, Q_{k, 1}, Q_{k, 2}, \ldots, Q_{k, N-1}$, we define

$$
\begin{aligned}
\Phi_{k} & \left(P_{k}, Q_{k, 1}, Q_{k, 2}, \ldots, Q_{k, N-1}\right) \\
= & \mathscr{A}_{k}^{\mathrm{T}} P_{k} \mathscr{A}_{k}-\mathscr{B}_{k}^{\mathrm{T}} P_{k} \mathscr{B}_{k} \\
& +\sum_{l=1}^{N-1}\left(\mathscr{C}_{k}^{\mathrm{T}} Q_{k, l} \mathscr{C}_{k}-\mathscr{D}_{k}^{\mathrm{T}} Q_{k, l} \mathscr{D}_{k}\right) .
\end{aligned}
$$

We are now ready to present the following result.

Theorem 1: Delay-difference equation (1) is strongly stable if there exist a positive integer $k$, a positive definite matrix $P_{k} \in \mathbf{S}^{k^{N-1} n}$ and $N-1$ matrices $Q_{k, l} \in \mathbf{S}^{k^{N-l}(k+1)^{l-1} n}, l=1,2, \ldots, N-1$ such that

$$
\Phi_{k}\left(P_{k}, Q_{k, 1}, Q_{k, 2}, \ldots, Q_{k, N-1}\right)<0_{(k+1)^{N-1} n} .
$$

Moreover, if delay-difference equation (1) is strongly stable, then there exist an integer $k^{*}$, a positive definite matrix $P_{k} \in \mathbf{S}^{k^{N-1} n}$ and $N-1$ matrices $Q_{k, l} \in \mathbf{S}^{k^{N-l}(k+1)^{l-1} n}, l=1,2, \ldots, N-1$ such that (9) is solvable for any $k \geq k^{*}$.

Proof: Sufficiency. Assume that (9) holds. By (3) and (4), we have

$$
L_{k} z^{[k+1]}=z^{[k]}, R_{k} z^{[k+1]}=z z^{[k]} .
$$

By multiplying both sides of (9), on the left by $\tilde{z}_{k}$, with $\left|z_{i}\right|=$ $1, i=1,2, \ldots, N-1$, and on the right by its conjugate-transpose, we obtain from (10) that

$$
0>A^{\mathrm{H}}(z) P(z) A(z)-P(z)+\sum_{l=1}^{N-1}\left(1-\left|z_{l}\right|^{2}\right) \tilde{z}_{k, l}^{\star \mathrm{H}} Q_{k, l} \tilde{z}_{k, l}^{\star},
$$

where $\tilde{z}_{k, l}^{\star}=z_{N-1}^{[k]} \otimes \cdots \otimes z_{l}^{[k]} \otimes z_{l-1}^{[k+1]} \otimes \cdots \otimes z_{1}^{[k+1]} \otimes I_{n}$. Since $\left|z_{i}\right|=1, i=1,2, \ldots, N-1$, it follows from (11) that (6) is satisfied.

Necessity. First, we show that if $A(z)$ is Schur stable for all $z_{i} \in \mathbf{C}$ with $\left|z_{i}\right|=1, i=1,2, \ldots, N-1$, then there exists a PPDQ function $x^{\mathrm{T}}(t) P(z) x(t)$ with $P_{k}>0$ such that

$$
A^{\mathrm{H}}(z) P(z) A(z)-P(z)<0
$$

for all $z_{i} \in \mathbf{C}$ with $\left|z_{i}\right|=1, i=1,2, \ldots, N-1$. When $A(z)$ is Schur stable for any $z_{i} \in \mathbf{C}$ with $\left|z_{i}\right|=1, i=1,2, \ldots, N-1$, the solution of the parameter-dependent Lyapunov equation

$$
A^{\mathrm{H}}(z) P(z) A(z)-P(z)=-I_{n},
$$


is analytic in $z_{i}$ and its conjugate $\bar{z}_{i}, i=1,2, \ldots, N-1$. The explicit solution of (13) is given by

$$
P(z)=\sum_{k=1}^{\infty}\left(A^{\mathrm{H}}(z)\right)^{k-1}(A(z))^{k-1} .
$$

Let $M_{1}, M_{2}, \ldots, M_{k}$ be some matrices with appropriate dimensions such that $M_{1} \tilde{z}_{1}=I, M_{2} \tilde{z}_{2}=A(z), \ldots, M_{k} \tilde{z}_{k}=(A(z))^{k-1}$. Then

$$
P(z)=\lim _{k \rightarrow \infty} \sum_{j=1}^{k}\left(\tilde{z}_{j}^{\mathrm{H}} M_{j}^{\mathrm{H}} M_{j} \tilde{z}_{j}\right)
$$

with uniform convergence for all $z_{i} \in \mathbf{C}$ with $\left|z_{i}\right|=1, i=$ $1,2, \ldots, N-1$. From the definition of $\tilde{z}_{k}$ and equation (4), it can be concluded that there exist non-zero constant matrices $G_{j, k} \in$ $\mathbf{C}^{j^{(N-1)} n \times k^{(N-1)} n}$ such that

$$
\tilde{z}_{j}=G_{j, k} \tilde{z}_{k}, 1 \leq j \leq k, j \in \mathbf{Z}^{+}, k \in \mathbf{Z}^{+} .
$$

Substituting (16) into (15), it can be obtained that

$$
\begin{aligned}
P(z) & =\lim _{k \rightarrow \infty} \sum_{j=1}^{k} \tilde{z}_{k}^{\mathrm{H}}\left(G_{j, k}^{\mathrm{H}} M_{j}^{\mathrm{H}} M_{j} G_{j, k}\right) \tilde{z}_{k} \\
& =\lim _{k \rightarrow \infty} \tilde{z}_{k}^{\mathrm{H}} \mathscr{P}_{k} \tilde{z}_{k},
\end{aligned}
$$

where

$$
\mathscr{P}_{k}=\sum_{j=1}^{k}\left(M_{j} G_{j, k}\right)^{\mathrm{H}}\left(M_{j} G_{j, k}\right) .
$$

This implies that, for large enough $k, \tilde{z}_{k}^{\mathrm{H}} \mathscr{P}_{k} \tilde{z}_{k}$ solves inequality (12) for all $z_{i} \in \mathbf{C}$ with $\left|z_{i}\right|=1, i=1,2, \ldots, N-1$. However, matrices $\mathscr{P}_{k}$ are only guaranteed to be semi-definite (except for $\mathscr{P}_{1}=P(0)>0$ ). To overcome this problem, we can use similar arguments as in [4]. We specify $P_{k}$ as

$$
P_{k} \triangleq \sum_{i_{1}, \ldots, i_{m}=0}^{k-1} F_{k} \mathscr{P}_{k} F_{k}^{\mathrm{T}},
$$

where $F_{k}=F_{k}^{i_{m}} \otimes \cdots \otimes F_{k}^{i_{1}} \otimes I_{n}$ and

$$
F_{k}^{i \mathrm{~T}}=\left[\begin{array}{cc}
0_{(k-i) \times i} & I_{k-i} \\
0_{i} & 0_{i \times(k-i)}
\end{array}\right], i \in \mathbf{Z}, \quad 0 \leq i \leq k-1 .
$$

Then by applying Lemma 7.1 in [4], we conclude that, for large enough $k$, matrix function (5), with $P_{k}$ given by (17), satisfies

$$
A^{\mathrm{H}}(z) P(z) A(z)-P(z)<0
$$

for any $z_{i} \in \mathbf{C}$ with $\left|z_{i}\right|=1, i=1,2, \ldots, N-1$. Second, we prove that the property that (19) is satisfied by (5) and (19) implies (9). Notice that, for any matrix $U \in \mathbf{C}^{p \times q}$ and any $u \in \mathbf{C}$, we have the identity [4]

$$
\left(u^{[k]} \otimes I_{p}\right) U=\left(I_{k} \otimes U\right)\left(u^{[k]} \otimes I_{q}\right) .
$$

By this identity and substituting (5) into (19) we obtain

$$
\left(\left(I_{k^{N-1}} \otimes A(z)\right) \tilde{z}_{k}\right)^{\mathrm{H}} P_{k}\left(\left(I_{k^{N-1}} \otimes A(z)\right) \tilde{z}_{k}\right)-\tilde{z}_{k}^{\mathrm{H}} P_{k} \tilde{z}_{k}<0 .
$$

Due to the identities

$$
\begin{aligned}
& \left(I_{k^{N-1}} \otimes A(z)\right) \tilde{z}_{k} \\
& =\left(I_{k^{N-1}} \otimes A_{1}\right) \tilde{z}_{k}+\left(I_{k^{N-1}} \otimes A_{2}\right)\left(z_{N-1}^{[k]} \otimes \cdots \otimes z_{1} z_{1}^{[k]} \otimes I_{n}\right) \\
& \quad+\cdots+\left(I_{k^{N-1}} \otimes A_{N}\right)\left(z_{N-1} z_{N-1}^{[k]} \otimes \cdots \otimes z_{1}^{[k]} \otimes I_{n}\right) \\
& =\mathscr{A}_{k}\left(z_{N-1}^{[k+1]} \otimes \cdots \otimes z_{1}^{[k+1]} \otimes I_{n}\right)
\end{aligned}
$$

and

$$
\tilde{z}_{k}=\left(L_{k}^{(N-1) \otimes} \otimes I_{n}\right)\left(z_{N-1}^{[k+1]} \otimes \cdots \otimes z_{1}^{[k+1]} \otimes I_{n}\right),
$$

inequality (21) can be rewritten as

$$
\tilde{z}_{k+1}^{\mathrm{H}}\left(\mathscr{A}_{k}^{\mathrm{T}} P_{k} \mathscr{A}_{k}-\mathscr{B}_{k}^{\mathrm{T}} P_{k} \mathscr{B}_{k}\right) \tilde{z}_{k+1}<0
$$

where $\tilde{z}_{k+1}=z_{N-1}^{[k+1]} \otimes \cdots \otimes z_{1}^{[k+1]} \otimes I_{n}$.

For $i \in\{0,1, \ldots, N-1\}$, we define the property $\left(\mathcal{P}_{i}\right)$ as follows: $\exists k \in \mathbf{Z}^{+}, \exists P_{k} \in \mathbf{S}^{k^{N-1} n}, P_{k}>0, \exists Q_{k, l} \in \mathbf{S}^{k^{N-l}(k+1)^{l-1} n}, l=$ $1,2, \ldots, i$, such that

$$
\begin{aligned}
0_{(k+1)^{i} n}> & \tilde{z}_{k, i}^{\dagger \mathrm{H}}\left(\mathscr{A}_{k}^{\mathrm{T}} P_{k} \mathscr{A}_{k}-\mathscr{B}_{k}^{\mathrm{T}} P_{k} \mathscr{B}_{k}\right. \\
& \left.+\sum_{l=1}^{i} \mathscr{C}_{k}^{\mathrm{T}} Q_{k, l} \mathscr{C}_{k}-\sum_{l=1}^{i} \mathscr{D}_{k}^{\mathrm{T}} Q_{k, l} \mathscr{D}_{k}\right) \tilde{z}_{k, i}^{\dagger},
\end{aligned}
$$

where $\tilde{z}_{k, i}^{\dagger}=z_{N-1}^{[k+1]} \otimes \cdots \otimes z_{1+i}^{[k+1]} \otimes I_{(k+1)^{i} n}$ for all $\left(z_{i+1}, \ldots, z_{N-1}\right) \in \mathbf{C}^{N-1-i}$ with $\left|z_{i+1}\right|=\cdots=\left|z_{N-1}\right|=1$. Clearly, Property $\left(\mathcal{P}_{0}\right)$ means that $\exists k \in \mathbf{Z}^{+}, \exists P_{k} \in \mathbf{S}^{k^{N-1} n}, P_{k}>0$ such that

$$
\tilde{z}_{k+1}^{\mathrm{H}}\left(\mathscr{A}_{k}^{\mathrm{T}} P_{k} \mathscr{A}_{k}-\mathscr{B}_{k}^{\mathrm{T}} P_{k} \mathscr{B}_{k}\right) \tilde{z}_{k+1}<0,
$$

which is equivalent to (22). At the same time, Property $\left(\mathcal{P}_{N-1}\right)$ corresponds to the condition in Theorem 1. By Lemma 7.2 in [4], it can be concluded that $\left(\mathcal{P}_{i}\right) \Leftrightarrow\left(\mathcal{P}_{i+1}\right)$ for any $i=0,1, \ldots, N-2$. The proof is completed.

The proposed LMI condition in Theorem 1 involves matrices that are affine functions of $\left(A_{1}, A_{2}, \ldots, A_{N}\right)$. This property is very helpful for studying robust strong stability problem of delaydifference equations with norm bounded uncertainties on the coefficient matrices, which we will address in Section III.

For the special case $N=2$, delay-difference equation (1) can be written as

$$
x(t)=A_{1} x\left(t-r_{1}\right)+A_{2} x\left(t-r_{2}\right) .
$$

Applying Theorem 1 to this special case leads to corollary 1 .

Corollary 1: Delay-difference equation (24) is strongly stable if there exist a positive integer $k$, a positive definite matrix $P_{k} \in \mathbf{S}^{k n}$ and a matrix $Q_{k, 1} \in \mathbf{S}^{k n}$ such that

$$
\begin{aligned}
& \left(L_{k} \otimes A_{1}+R_{k} \otimes A_{2}\right)^{\mathrm{T}} P_{k}\left(L_{k} \otimes A_{1}+R_{k} \otimes A_{2}\right) \\
& +\left(L_{k} \otimes I_{n}\right)^{\mathrm{T}}\left(Q_{k, 1}-P_{k}\right)\left(L_{k} \otimes I_{n}\right) \\
& -\left(R_{k} \otimes I_{n}\right)^{\mathrm{T}} Q_{k, 1}\left(R_{k} \otimes I_{n}\right)<0 .
\end{aligned}
$$

Moreover, if equation (24) is strongly stable, then there exist an integer $k^{*}$, a positive definite matrix $P_{k}$, a symmetric matrix $Q_{k, 1}$ such that (9) is solvable for any $k \geq k^{*}$.

By a congruence transformation, (25) is equivalent to

$$
\begin{aligned}
& \left(R_{k} \otimes A_{1}+L_{k} \otimes A_{2}\right)^{\mathrm{T}} P_{k}\left(R_{k} \otimes A_{1}+L_{k} \otimes A_{2}\right) \\
& +\left(R_{k} \otimes I_{n}\right)^{\mathrm{T}}\left(Q_{k, 1}-P_{k}\right)\left(R_{k} \otimes I_{n}\right) \\
& -\left(L_{k} \otimes I_{n}\right)^{\mathrm{T}} Q_{k, 1}\left(L_{k} \otimes I_{n}\right)<0 .
\end{aligned}
$$

Moreover, if $Q_{k, 1}$ is assumed to be a positive definite matrix, then (26) reduces to the LMI in Theorem 1 of [41].

Remark 1: The strong stability of coupled delay-difference equations in the form of [20],

$$
y_{i}(t)=\sum_{j=1}^{N} D_{i j} y_{j}\left(t-r_{i}\right), i=1,2, \ldots, N,
$$

where $D_{i j} \in \mathbf{R}^{m_{i} \times m_{j}}, m_{i}, m_{j} \in \mathbf{Z}^{+}$, can also be assessed by Theorem 1. As discussed in [20], equation (27) is exponentially stable 
for all $\left(r_{1}, r_{2}, \ldots, r_{N}\right)$ satisfying $r_{i}>0, i=1,2, \ldots, N$ if and only if

$$
\sup _{\left(\theta_{1}, \theta_{2}, \ldots, \theta_{N}\right) \in[0,2 \pi]^{N}} \rho\left(D E\left(\mathrm{j} \theta_{1}, \mathrm{j} \theta_{2}, \ldots, \mathrm{j} \theta_{N}\right)\right)<1,
$$

where

$$
E\left(\mathrm{j} \theta_{1}, \ldots, \mathrm{j} \theta_{N}\right)=\operatorname{diag}\left\{\mathrm{e}^{-\mathrm{j} \theta_{1}} I_{m_{1}}, \ldots, \mathrm{e}^{-\mathrm{j} \theta_{N}} I_{m_{N}}\right\}
$$

and

$$
D=\left[\begin{array}{cccc}
D_{11} & D_{12} & \cdots & D_{1 N} \\
D_{21} & D_{22} & \cdots & D_{2 N} \\
\vdots & \vdots & \ddots & \vdots \\
D_{N 1} & D_{N 2} & \cdots & D_{N N}
\end{array}\right]
$$

By simple computations, one has

$$
\begin{aligned}
1> & \sup _{\left(\theta_{1}, \theta_{2}, \ldots, \theta_{N}\right) \in[0,2 \pi]^{N}} \rho\left(D E\left(\mathrm{j} \theta_{1}, \mathrm{j} \theta_{2}, \ldots, \mathrm{j} \theta_{N}\right)\right) \\
& =\sup _{\left(\theta_{1}, \ldots, \theta_{N}\right) \in[0,2 \pi]^{N}} \rho\left(\mathrm{e}^{-\mathrm{j} \theta_{1}} \bar{D}_{1}+\mathrm{e}^{-\mathrm{j} \theta_{2}} \bar{D}_{2}+\cdots+\mathrm{e}^{-\mathrm{j} \theta_{N}} \bar{D}_{N}\right),
\end{aligned}
$$

where

$$
\begin{aligned}
\bar{D}_{1}= & D \operatorname{diag}\left\{I_{m_{1}}, 0_{m_{2}}, \ldots, 0_{m_{N}}\right\}, \\
\bar{D}_{2}= & D \operatorname{diag}\left\{0_{m_{1}}, I_{m_{2}}, 0_{m_{3}}, \ldots, 0_{m_{N}}\right\}, \\
& \vdots \\
\bar{D}_{N}= & D \operatorname{diag}\left\{0_{m_{1}}, \ldots, 0_{m_{N-1}}, I_{m_{N}}\right\} .
\end{aligned}
$$

Remark 2: Following the idea in [29], the convergence rate analysis of (1) can be performed by replacing $A_{i}, i=1,2, \ldots, N$, in Theorem 1 with $\mathrm{e}^{\mu r_{i}} A_{i}, i=1,2, \ldots, N$, where $\mu>0$ is a constant denoting a guaranteed convergence rate.

Remark 3: Determining the order $k^{*}$ in Theorem 1 is a very difficult problem, and an answer even for special cases is hard to obtain, let it be a tractable bound. Following the proof of Theorem 1, a first question in the quantification of $k^{*}$ is the following: given that for a strongly stable system series (15) satisfies

$$
A^{H}(z) P(z) A(z)-P(z)=-I
$$

for all $\left(z_{1}, z_{2}, . ., z_{N-1}\right) \in \mathbf{C}^{N-1}$ with $\left|z_{i}\right|=1, i=1, \ldots, N-1$, what is then the minimal number of terms in a truncation of the series in order to preserve the negative definiteness of the left hand side of (28). This is a very difficult question from multi-variable approximation theory, and the answer is much problem dependent. The second question, of the same level of difficulty, is to quantify the convergence property in the proof of Lemma 7.1 of [4] on which ours relies.

Remark 4: For a given $k$, the computation complexity of the condition (9) depends polynomially on the state dimension $n$, exponentially on the number $N$ of delays. Obviously, the computation burden grows rapidly with the number of delays and state dimension. Thus this approach is more suitable for system with small state dimension or small number of delays.

\section{B. Interpretations and Relationships}

We first present a time-domain interpretation of Theorem 1 in terms of a quadratic integral Lyapunov functional.

For all nonnegative numbers $\bar{r}_{i}=r_{i+1}-r_{1}, i=1,2, \ldots, N-$ 1 , we define a relevant operator $\Delta$ on the set of functions $x$ by $\left(\Delta_{\bar{r}_{i}} x\right)(t)=x\left(t-\bar{r}_{i}\right)$. For any $k_{N-1}, \ldots, k_{1} \in \mathbf{Z}^{+}$, we define the augmented state variable $X_{k_{N-1}, \ldots, k_{1}}(t) \in \mathbf{R}^{k_{N-1} \ldots k_{1} n}$ as

$$
X_{k_{N-1}, \ldots, k_{1}}(t)=\left(\Delta_{\bar{r}_{N-1}}^{\left[k_{N-1}\right]} \otimes \cdots \otimes \Delta_{\bar{r}_{1}}^{\left[k_{1}\right]}\right) x(t),
$$

where $\Delta_{\bar{r}_{i}}^{\left[k_{i}\right]}=\left[1, \Delta_{\bar{r}_{i}}, \ldots, \Delta_{\bar{r}_{i}}^{k_{i}-1}\right]^{\mathrm{T}}$ corresponds to (4).

We choose the quadratic integral Lyapunov functional,

$$
\begin{aligned}
V & \left(X_{k, k+1, \ldots, k+1}(t)\right) \\
= & \int_{t-r_{1}}^{t} X_{k, \ldots, k}^{\mathrm{T}}(s) P_{k} X_{k, \ldots, k}(s) \mathrm{d} s \\
& +\int_{t-r_{2}}^{t-r_{1}} X_{k, \ldots, k}^{\mathrm{T}}(s) Q_{k, 1} X_{k, \ldots, k}(s) \mathrm{d} s \\
& +\int_{t-r_{3}}^{t-r_{1}} X_{k, \ldots, k, k+1}^{\mathrm{T}}(s) Q_{k, 2} X_{k, \ldots, k, k+1}(s) \mathrm{d} s+\cdots \\
& +\int_{t-r_{N}}^{t-r_{1}} X_{k, k+1, \ldots, k+1}^{\mathrm{T}}(s) Q_{k, N-1} X_{k, k+1, \ldots, k+1}(s) \mathrm{d} s
\end{aligned}
$$

where $Q_{k, j}>0$ if $r_{j+1} \geq r_{1}$ and $Q_{k, j}<0$ otherwise. Its timederivative along the solutions of delay-difference equation (1) is given by

$$
\begin{aligned}
& \frac{\mathrm{d}}{\mathrm{d} t} V\left(X_{k, k+1, \ldots, k+1}(t)\right) \\
& =X_{k, \ldots, k}^{\mathrm{T}}(t) P_{k} X_{k, \ldots, k}(t)-X_{k, \ldots, k}^{\mathrm{T}}\left(t-r_{1}\right) P_{k} X_{k, \ldots, k}\left(t-r_{1}\right) \\
& \quad+\cdots-X_{k, k+1, \ldots, k+1}^{\mathrm{T}}\left(t-r_{N}\right) Q_{k, N-1} X_{k, k+1, \ldots, k+1}\left(t-r_{N}\right) .
\end{aligned}
$$

By (1), it can be obtained that

$$
\begin{aligned}
X_{k, \ldots, k}(t)= & \left(I_{k^{N-1}} \otimes A_{1}\right) X_{k, \ldots, k}\left(t-r_{1}\right)+\cdots \\
& +\left(I_{k^{N-1}} \otimes A_{N}\right) X_{k, \ldots, k}\left(t-r_{N}\right) .
\end{aligned}
$$

Using the fact that $L_{k}\left(\Delta_{\bar{r}_{i}}^{[k+1]} x\right)=\Delta_{\bar{r}_{i}}^{[k]} x$ and $R_{k}\left(\Delta_{\bar{r}_{i}}^{[k+1]} x\right)=$ $\Delta\left(\Delta_{\bar{r}_{i}}^{[k]} x\right)$, we obtain

$$
\begin{aligned}
X_{k, \ldots, k}\left(t-r_{1}\right) & =\left(L_{k}^{(N-1) \otimes} \otimes I_{n}\right) \mathscr{X}_{k+1}, \\
X_{k, \ldots, k}\left(t-r_{2}\right) & =\left(L_{k}^{(N-2) \otimes} \otimes R_{k} \otimes I_{n}\right) \mathscr{X}_{k+1}, \\
\vdots & \\
X_{k, \ldots, k}\left(t-r_{N}\right) & =\left(R_{k} \otimes L_{k}^{(N-2) \otimes} \otimes I_{n}\right) \mathscr{X}_{k+1}, \\
X_{k, \ldots, k, k+1}\left(t-r_{1}\right) & =\left(L_{k}^{(N-2) \otimes} \otimes I_{(k+1) n}\right) \mathscr{X}_{k+1}, \\
\vdots & \\
X_{k, k+1, \ldots, k+1}\left(t-r_{1}\right) & =\left(L_{k} \otimes I_{(k+1)}{ }^{N-2} n\right) \mathscr{X}_{k+1},
\end{aligned}
$$

and

$$
\begin{aligned}
X_{k, \ldots, k, k+1}\left(t-r_{3}\right) & =\left(L_{k}^{(N-3) \otimes} \otimes R_{k} \otimes I_{(k+1) n}\right) \mathscr{X}_{k+1}, \\
\vdots & \\
X_{k, k+1, \ldots, k+1}\left(t-r_{N}\right) & =\left(R_{k} \otimes I_{(k+1)^{N-2} n}\right) \mathscr{X}_{k+1},
\end{aligned}
$$

where

$$
\mathscr{X}_{k+1}=X_{k+1, k+1, \ldots, k+1}\left(t-r_{1}\right) .
$$

For notation simplicity, we denote

$$
\Phi_{k}=\Phi_{k}\left(P_{k}, Q_{k, 1}, Q_{k, 2}, \ldots, Q_{k, N-1}\right) .
$$

By (31), (32) and (33), it can be concluded that

$$
\frac{\mathrm{d}}{\mathrm{d} t} V\left(X_{k, k+1, \ldots, k+1}(t)\right)=\mathscr{X}_{k+1}^{\mathrm{T}} \Phi_{k} \mathscr{X}_{k+1} .
$$

We conclude that LMI (9) in Theorem 1 is related to searching for an integral Lyapunov functional in the form of (29). Note that there is no assumption on the positivity of $Q_{k, l}, l=1,2, \ldots, N-1$. Of course, if the matrices $Q_{k, l}$ in the LMI (9) are further assumed to be positive definite, the strong stability of (1) can certainly be 
guaranteed. Experiments with many numerical examples indicate that the additional assumption that matrices $Q_{k, l}, l=1,2, \ldots, N-1$ are positive definite will not introduce conservatism into the condition. Note that in [5], a quadratic LKF was shown to be associated with the established LMIs in Theorem 5. It should be mentioned that (29) is different from the LKF constructed in [5].

Next, we make a comparison with an existing result. Denoting by $A_{\mathrm{c}}$ the $n N \times n N$ block-companion matrix

$$
A_{\mathrm{c}}=\left[\begin{array}{cccc}
A_{1} & A_{2} & \cdots & A_{N} \\
I_{n} & & & \\
& \ddots & & \\
& & I_{n} & 0_{n}
\end{array}\right],
$$

we state a stability condition for (1) from [12], which is equivalent to a stability condition found in [18].

Lemma 1: [12] Delay-difference equation (1) is strongly stable if there exist $n \times n$ symmetric positive definite matrices $X_{1}, X_{2}, \ldots, X_{N} \in \mathbf{R}^{n \times n}$ such that the following LMI is satisfied,

$$
\Psi_{1}\left(X_{1}, X_{2}, \ldots, X_{N}\right) \triangleq A_{\mathrm{c}}^{\mathrm{T}} X_{d} A_{\mathrm{c}}-X_{d}<0,
$$

where $X_{d}=\operatorname{diag}\left\{X_{1}, X_{2}, \ldots, X_{N}\right\}$.

Proof: For future use, we provide a brief proof here. Denote $\Psi_{1}=\Psi_{1}\left(X_{1}, X_{2}, \ldots, X_{N}\right)$. Choose the integral Lyapunov functional

$$
\begin{aligned}
W_{1}\left(x_{t}\right)= & \int_{t-r_{1}}^{t} x^{\mathrm{T}}(s) X_{1} x(s) \mathrm{d} s \\
& +\sum_{i=2}^{N} \int_{t-r_{i}}^{t-r_{i-1}} x^{\mathrm{T}}(s) X_{i} x(s) \mathrm{d} s,
\end{aligned}
$$

which is such that

$$
\frac{\mathrm{d}}{\mathrm{d} t} W_{1}\left(x_{t}\right)=\mathscr{X}_{1}^{\mathrm{T}} \Psi_{1} \mathscr{X}_{1}
$$

where $\mathscr{X}_{1}=\left[\begin{array}{llll}x\left(t-r_{1}\right)^{\mathrm{T}} & x\left(t-r_{2}\right)^{\mathrm{T}} & \cdots & x\left(t-r_{N}\right)^{\mathrm{T}}\end{array}\right]^{\mathrm{T}}$. Since $\Psi_{1}<0$, delay-difference equation (1) is strongly stable by the Lyapunov theorem [12].

We are now ready to relate (35) with Theorem 1 . If we set $k=1$ in Theorem 1 and denote $P_{1}=X_{1}$,

$$
\begin{aligned}
Q_{1,1} & =\operatorname{diag}\left\{X_{2}-X_{3}\right\} \in \mathbf{R}^{n \times n}, \\
Q_{1,2} & =\operatorname{diag}\left\{X_{3}-X_{4}, 0_{n}\right\} \in \mathbf{R}^{2 n \times 2 n}, \\
& \vdots \\
Q_{1, N-1} & =\operatorname{diag}\left\{X_{N}, 0_{n}, \ldots, 0_{n}\right\} \in \mathbf{R}^{2^{N-2} n \times 2^{N-2} n},
\end{aligned}
$$

we obtain the following result.

Lemma 2: Let $\Phi_{1}\left(P_{1}, Q_{1,1}, \ldots, Q_{1, N-1}\right)$ be defined in (8) and $\Psi_{1}\left(X_{1}, \ldots, X_{N}\right)$ be defined in (35). Then, for $k=1$, it holds that

$$
E_{N} \Phi_{1} E_{N}^{\mathrm{T}}=\Psi_{1}
$$

where

$$
E_{N}=\left[\begin{array}{ccc}
I_{n} & 0_{n \times\left(2^{N-1}-1\right) n} & \\
0_{n \times 2^{0} n} & I_{n} & 0_{n \times\left(2^{N-1}-2^{0}-1\right) n} \\
0_{n \times 2^{1} n} & I_{n} & 0_{n \times\left(2^{N-1}-2^{1}-1\right) n} \\
\vdots & & \vdots \\
0_{n \times 2^{N-2} n} & I_{n} & 0_{n \times\left(2^{N-1}-2^{N-2}-1\right) n}
\end{array}\right] .
$$

Proof: Let $k=1$. Then it follows from (29) that

$$
\begin{aligned}
V & \left(X_{1,2, \ldots, 2}(t)\right) \\
= & \int_{t-r_{1}}^{t} X_{1, \ldots, 1}^{\mathrm{T}}(s) P_{1} X_{1, \ldots, 1}(s) \mathrm{d} s \\
& +\int_{t-r_{2}}^{t-r_{1}} X_{1, \ldots, 1}^{\mathrm{T}}(s) Q_{1,1} X_{1, \ldots, 1}(s) \mathrm{d} s+\cdots \\
& +\int_{t-r_{N}}^{t-r_{1}} X_{1,2, \ldots, 2}^{T}(s) Q_{1, N-1} X_{1,2, \ldots, 2}(s) \mathrm{d} s,
\end{aligned}
$$

and from (30) that

$$
\begin{aligned}
& \frac{\mathrm{d}}{\mathrm{d} t} V\left(X_{1,2, \ldots, 2}(t)\right) \\
& =X_{1, \ldots, 1}^{\mathrm{T}}(t) P_{1} X_{1, \ldots, 1}(t)-X_{1, \ldots, 1}^{\mathrm{T}}\left(t-r_{1}\right) P_{1} X_{1, \ldots, 1}\left(t-r_{1}\right) \\
& \quad+\cdots-X_{1,2, \ldots, 2}^{\mathrm{T}}\left(t-r_{N}\right) Q_{1, N-1} X_{1,2, \ldots, 2}\left(t-r_{N}\right) \\
& =\mathscr{X}_{2}^{\mathrm{T}} \Phi_{1} \mathscr{X}_{2}=\mathscr{X}_{1}^{\mathrm{T}} E_{N} \Phi_{1} E_{N}^{\mathrm{T}} \mathscr{X}_{1} .
\end{aligned}
$$

By comparing (36) and (37) with (39) and (40), respectively, we obtain (38). The proof is completed.

We conclude from (38) that Lemma 1 can be considered as a special case of Theorem 1. More precisely, it is shown that $\Psi_{1}$ is a projection of $\Phi_{1}$. So we have that $\Phi_{1}<0$ implies $\Psi_{1}<0$ but not necessarily the other way around.

Remark 5: Reference [41] shows that, for $N=2$, Theorem 1 with $k=2$ is equivalent to Theorem 4 in [14]. However, for $N \geq 3$, the relationship between Theorem 1 with $k=2$ and Theorem 4 in [14] is not clear, even though many numerical experiments show that Theorem 1 with $k=2$ is as good as or better than Theorem 4 in [14].

\section{RobUSt Strong StABILITY}

In this section we assume that delay-difference equation (1) is perturbed to

$$
x(t)=\sum_{i=1}^{N}\left(A_{i}+\Delta A_{i}\right) x\left(t-r_{i}\right), t \geq 0,
$$

where $A_{i} \in \mathbf{R}^{n \times n}, i=1, \ldots, N$, are the same as in (1) and

$\left[\begin{array}{llll}\Delta A_{1} & \Delta A_{2} & \cdots & \Delta A_{N}\end{array}\right]=\bar{E} F\left[\begin{array}{llll}\bar{A}_{1} & \bar{A}_{2} & \cdots & \bar{A}_{N}\end{array}\right]$,

where $\bar{E} \in \mathbf{R}^{n \times p}, \bar{A}_{i} \in \mathbf{R}^{q \times n}, i=1, \ldots, N$, are known matrices, and $F \in \mathbf{R}^{p \times q}$ denotes the a norm bounded (time-varying) that satisfies the inequality

$$
F^{\mathrm{T}} F \leq I_{q}
$$

In what follows, we investigate the strong stability of perturbed delaydifference equation (41). First we present a condition in terms of structured singular values, which is in the spirit of criterion (2). Subsequently, we derive LMI conditions based on Theorem 1.

\section{A. A Necessary and Sufficient Condition in Terms of the Structured Singular Values}

In this subsection, a necessary and sufficient condition for the robust strong stability of (41) is presented in terms of the real structured singular value, which is defined by

$$
\mu_{\mathbf{R}}(M) \triangleq \begin{cases}0, & \text { if } \operatorname{det}\left(I_{q}-M F\right) \neq 0 \text { for all } F \in \mathbf{R}^{p \times q}, \\ \mu_{F}^{-1}, & \text { otherwise, }\end{cases}
$$

where

$$
\mu_{F}=\inf _{F \in \mathbf{R}^{p \times q}}\left\{\|F\|_{2}: \operatorname{det}\left(I_{q}-M F\right)=0\right\}
$$

with $M \in \mathbf{C}^{q \times p}$. In [31] a computational expression is provided,

$$
\mu_{\mathbf{R}}(M)=\inf _{\gamma \in(0,1]} \sigma_{2}\left[\begin{array}{rr}
\operatorname{Re}(M) & -\gamma \operatorname{Im}(M) \\
\gamma^{-1} \operatorname{Im}(M) & \operatorname{Re}(M)
\end{array}\right],
$$


where $\sigma_{2}(M)$ is the second (largest) singular value of $M$.

Denoting

$$
A\left(\theta_{1}, \ldots, \theta_{N}\right)=\sum_{i=1}^{N} A_{i} \mathrm{e}^{\mathrm{j} \theta_{i}}, \bar{A}\left(\theta_{1}, \ldots, \theta_{N}\right)=\sum_{i=1}^{N} \bar{A}_{i} \mathrm{e}^{\mathrm{j} \theta_{i}},
$$

we can state the following result.

Theorem 2: Assume that delay-difference equation (1) is strongly stable. Then the perturbed delay-difference equation (41) is robustly strongly stable if and only if

$$
\max _{\left(\theta_{1}, \ldots, \theta_{N}\right) \in[0,2 \pi]^{N}} \mu_{\mathbf{R}}\left(\bar{A}\left(\theta_{1}, \ldots, \theta_{N}\right) \Xi^{-1} \bar{E}\right)<1,
$$

where

$$
\Xi=I_{n}-A\left(\theta_{1}, \ldots, \theta_{N}\right) .
$$

Proof: By virtue of (2), perturbed delay-difference equation (41) is strongly stable if and only if

$$
\max _{\left(\theta_{1}, \ldots, \theta_{N}\right) \in[0,2 \pi]^{N}} \rho\left(A\left(\theta_{1}, \ldots, \theta_{N}\right)+\bar{E} F \bar{A}\left(\theta_{1}, \ldots, \theta_{N}\right)\right)<1 .
$$

The unperturbed delay-difference equation ((41) with $F=0)$ is strongly stable. Thus, we can can define the real distance to instability (also called real stability radius) associated with

$d_{\mathrm{INS}}^{\mathbf{R}}= \begin{cases}+\infty, & \text { if }(41) \text { is strongly stable for all } F \in \mathbf{R}^{p \times q}, \\ d_{F}, & \text { otherwise, }\end{cases}$ where $d_{F}=\inf _{F \in \mathbf{R}^{p \times q}}\left\{\|F\|_{2}:\right.$ (41) is not strongly stable $\}$.

Suppose that, for some fixed $F$, the perturbed delay-difference equation (41) is at the border of strong stability (more precisely, it is strongly stable for $\alpha F$ with $\alpha \in[0,1)$ but not for $\alpha=1)$. Then we must have

$$
\operatorname{det}\left(\Xi-\bar{E} F \bar{A}\left(\theta_{1}, \ldots, \theta_{N}\right)\right)=0
$$

for some $\left(\theta_{1}, \ldots, \theta_{N}\right)$, which can be rephrased as

$$
\operatorname{det}\left(I_{q}-G\left(\theta_{1}, \ldots, \theta_{N}\right) F\right)=0,
$$

where

$$
G\left(\theta_{1}, \ldots, \theta_{N}\right)=\bar{A}\left(\theta_{1}, \ldots, \theta_{N}\right) \Xi^{-1} \bar{E} .
$$

It follows that $d_{\mathrm{INS}}^{\mathrm{R}}$ can be inferred from considering all solutions $\left(\theta_{1}, \theta_{2}, \ldots, \theta_{N}, F\right)$ of (44) and selecting the one with minimum $\|F\|_{2}$.

For any fixed $\left(\theta_{1}, \ldots, \theta_{N}\right)$, the smallest $F$ in spectral norm for which (44) is satisfied, has norm $\left(\mu_{\mathbf{R}}\left(G\left(\theta_{1}, \ldots, \theta_{N}\right)\right)\right)^{-1}$. Thus, the real distance to instability can be expressed as

$$
\begin{aligned}
d_{\mathrm{INS}}^{\mathbf{R}} & =\min _{\left(\theta_{1}, \ldots, \theta_{N}\right) \in[0,2 \pi]^{N}} \frac{1}{\mu_{\mathbf{R}}\left(G\left(\theta_{1}, \ldots, \theta_{N}\right)\right)} \\
& =\left(\max _{\left(\theta_{1}, \ldots, \theta_{N}\right) \in[0,2 \pi]^{N}} \mu_{\mathbf{R}}\left(G\left(\theta_{1}, \ldots, \theta_{N}\right)\right)\right)^{-1} .
\end{aligned}
$$

The proof is completed by noting that system (41), with $\|F\| \leq 1$, is robustly strongly stable if and only if $d_{\mathrm{INS}}^{\mathrm{R}}>1$.

Remark 6: Expression (44) is a standard form used in the structured singular value framework. As a consequence, one may impose any structure on $F$, and the corresponding stability radius can then be expressed in terms of the corresponding structured singular value. In case we just assume that $F \in \mathbf{C}^{p \times q}$, then we obtain the following expression for the complex stability radius,

$$
d_{\mathrm{INS}}^{\mathbf{C}}=\left(\max _{\left(\theta_{1}, \ldots, \theta_{N}\right) \in[0,2 \pi]^{N}} \sigma_{1}\left(G\left(\theta_{1}, \ldots, \theta_{N}\right)\right)\right)^{-1} \leq d_{\mathrm{INS}}^{\mathrm{R}},
$$

with $\sigma_{1}(M)$ denoting the largest singular value of $M$. with $\sigma_{1}(M)$ denoting the largest singular value of $M$. Note that the expression in the left can be interpreted as the reciprocal of the $\mathcal{H}_{\infty}$ norm of a $N$-dimensional discrete-time linear system.

\section{B. A Sufficient Condition in Terms of LMIs}

For $N=2$, the robust strong stability analysis problem has been studied in an LMI framework in the recent article [41]. In this subsection, we will extend the result in [41] to the general case where $N$ is any positive integer. For further usage, we denote $\overline{\mathscr{A}}_{k}=\mathscr{A}_{k}\left(\bar{A}_{1}, \bar{A}_{2}, \ldots, \bar{A}_{N}\right)$ and

$$
\Omega_{k}=-\mathscr{B}_{k}^{\mathrm{T}} P_{k} \mathscr{B}_{k}+\sum_{l=1}^{N-1}\left(\mathscr{C}_{k}^{\mathrm{T}} Q_{k, l} \mathscr{C}_{k}-\mathscr{D}_{k}^{\mathrm{T}} Q_{k, l} \mathscr{D}_{k}\right) .
$$

Theorem 3: The perturbed delay-difference equation (41) is strongly stable if there exist a positive integer $k$, a positive definite matrix $P_{k} \in \mathbf{S}^{k^{N-1} n}$, a positive definite matrix $S_{k} \in \mathbf{S}^{k^{N-1}}$ and $N-1$ matrices $Q_{k, l} \in \mathbf{S}^{k^{N-l}(k+1)^{l-1} n}, l=1,2, \ldots, N-1$ such that

$$
\left[\begin{array}{cc}
\Omega_{k}+\mathscr{A}_{k}^{\mathrm{T}} P_{k} \mathscr{A}_{k}+T & \mathscr{A}_{k}^{\mathrm{T}} P_{k} E_{k} \\
E_{k}^{\mathrm{T}} P_{k} \mathscr{A}_{k} & -\left(S_{k} \otimes I_{p}\right)+E_{k}^{\mathrm{T}} P_{k} E_{k}
\end{array}\right]<0,
$$

where

$$
T=\overline{\mathscr{A}}_{k}^{\mathrm{T}}\left(S_{k} \otimes I_{q}\right) \overline{\mathscr{A}}_{k}, E_{k}=\left(I_{k^{N-1}} \otimes \bar{E}\right) .
$$

Proof: Notice that

$$
\begin{aligned}
0> & {\left[\begin{array}{cc}
\Omega_{k}+T & 0_{(k+1)^{N-1} n \times k^{N-1} p} \\
0_{k^{N-1} p \times(k+1)^{N-1} n} & -\left(S_{k} \otimes I_{p}\right)
\end{array}\right] } \\
& +\left[\begin{array}{c}
\mathscr{A}_{k}^{\mathrm{T}} P_{k} \\
E_{k}^{\mathrm{T}} P_{k}
\end{array}\right] P_{k}^{-1}\left[\begin{array}{c}
\mathscr{A}_{k}^{\mathrm{T}} P_{k} \\
E_{k}^{\mathrm{T}} P_{k}
\end{array}\right]^{\mathrm{T}},
\end{aligned}
$$

which, by the Schur complement lemma, is equivalent to

$$
\left[\begin{array}{ccc}
\Omega_{k}+T & 0_{(k+1)^{N-1} n \times k^{N-1} p} & \mathscr{A}_{k}^{\mathrm{T}} P_{k} \\
0_{k^{N-1} p \times(k+1)^{N-1} n} & -\left(S_{k} \otimes I_{p}\right) & E_{k}^{\mathrm{T}} P_{k} \\
P_{k} \mathscr{A}_{k} & P_{k} E_{k} & -P_{k}
\end{array}\right]<0 .
$$

By a congruence transformation, the above inequality is equivalent to

$$
\left[\begin{array}{ccc}
\Omega_{k}+T & \mathscr{A}_{k}^{\mathrm{T}} P_{k} & 0_{(k+1)^{N-1} n \times k^{N-1} p} \\
P_{k} \mathscr{A}_{k} & -P_{k} & P_{k} E_{k} \\
0_{k^{N-1} p \times(k+1)^{N-1} n} & E_{k}^{\mathrm{T}} P_{k} & -\left(S_{k} \otimes I_{p}\right)
\end{array}\right]<0,
$$

which, by the Schur complement lemma, can be expressed as

$$
0>\Pi+H_{1}\left(S_{k}^{-1} \otimes I_{p}\right) H_{1}^{\mathrm{T}}+H_{2}^{\mathrm{T}}\left(S_{k} \otimes I_{q}\right) H_{2},
$$

where

$$
H_{1}=\left[\begin{array}{c}
0_{(k+1)^{N-1} n \times k^{N-1} p} \\
P_{k} E_{k}
\end{array}\right], H_{2}=\left[\begin{array}{c}
\overline{\mathscr{A}}_{k}^{\mathrm{T}} \\
0_{k^{N-1} n \times k^{N-1} q}
\end{array}\right]^{\mathrm{T}},
$$

and

$$
\Pi=\left[\begin{array}{cc}
\Omega_{k} & \mathscr{A}_{k}^{\mathrm{T}} P_{k} \\
P_{k} \mathscr{A}_{k} & -P_{k}
\end{array}\right]
$$

By (42), we have

$$
\Delta \overline{\mathscr{A}}_{k} \triangleq E_{k}\left(I_{k^{N-1}} \otimes F\right) \overline{\mathscr{A}}_{k}
$$

and using (43), we compute

$$
\left(I_{k^{N-1}} \otimes F^{\mathrm{T}}\right)\left(S_{k} \otimes I_{p}\right)\left(I_{k^{N-1}} \otimes F\right) \leq S_{k} \otimes I_{q} .
$$

Let $X, Y$ and $Q>0$ be some real matrices of appropriate dimensions. Then

$$
X Y+Y^{\mathrm{T}} X^{\mathrm{T}} \leq X Q X^{\mathrm{T}}+Y^{\mathrm{T}} Q^{-1} Y .
$$


From this result and from (46) it follows that

$$
\begin{aligned}
& {\left[\begin{array}{cc}
\Omega_{k} & \left(\mathscr{A}_{k}+\Delta \overline{\mathscr{A}}_{k}\right)^{\mathrm{T}} P_{k} \\
P_{k}\left(\mathscr{A}_{k}+\Delta \overline{\mathscr{A}}_{k}\right) & -P_{k}
\end{array}\right] } \\
= & \Pi+H_{1}\left(I_{k^{N-1}} \otimes F\right) H_{2}+H_{2}^{\mathrm{T}}\left(I_{k^{N-1}} \otimes F^{\mathrm{T}}\right) H_{1}^{\mathrm{T}} \\
\leq & \Pi+H_{1}\left(S_{k}^{-1} \otimes I_{p}\right) H_{1}^{\mathrm{T}}+H_{2}^{\mathrm{T}}\left(S_{k} \otimes I_{q}\right) H_{2} \\
< & 0 .
\end{aligned}
$$

By the Schur complement lemma, the above inequality is equivalent to

$$
0>\Omega_{k}+\left(\mathscr{A}_{k}+\Delta \overline{\mathscr{A}}_{k}\right)^{\mathrm{T}} P_{k}\left(\mathscr{A}_{k}+\Delta \overline{\mathscr{A}}_{k}\right) .
$$

From the result in Theorem 1, it can be concluded that perturbed delay-difference equation (41) is strongly stable. The proof is completed.

Remark 7: The proposed LMI condition can also be used for state feedback design. Details are omitted for space limitation.

\section{EXAMPLES}

In this section, we use three examples to show the effectiveness of the proposed results.

Example 1: Consider a delay-difference equation in the form of (1) with $N=3$, and

$$
\begin{gathered}
A_{1}=\frac{1}{\gamma_{0}}\left[\begin{array}{cccc}
-0.15 & 0 & 0.32 & 0 \\
0 & -0.07 & 0 & 0.05 \\
0.08 & 0 & 0.04 & 0 \\
0.2 & 0.03 & 0 & -0.13
\end{array}\right], \\
A_{2}=\frac{1}{\gamma_{0}}\left[\begin{array}{cccc}
-0.02 & 0.12 & 0 & 0.25 \\
0 & -0.05 & 0.04 & 0 \\
0 & 0.23 & 0 & -0.3 \\
0.19 & 0 & 0.28 & -0.09
\end{array}\right], \\
A_{3}=\frac{1}{\gamma_{0}}\left[\begin{array}{cccc}
0 & 0 & -0.03 & 0.14 \\
0.01 & -0.04 & 0 & 0 \\
0 & 0 & 0.09 & 0.26 \\
0.05 & -0.27 & -0.06 & 0
\end{array}\right],
\end{gathered}
$$

where $\gamma_{0} \in \mathbf{R}$ is a free parameter to be determined. We will use Theorem 1 to determine the minimum $\gamma_{0}$ such that system (1) is strongly stable. By a bisection search, we obtain that the minimum $\gamma_{0}$ by Theorem 1 with $k=2$ is 0.6028 , which is same as the $\gamma_{0}$, obtained in [25] and the value of $\gamma_{0}$ obtained from (2). This implies that Theorem 1 with $k=2$ is efficient for obtaining nonconservative stability conditions. In fact, a couple of numerical examples (with different coefficient matrices $\left(A_{1}, A_{2}, A_{3}\right)$ ) show that Theorem 1 with $k=2$ lead to nonconservative stability conditions.

Example 2: As an illustration of the applications of our results, we consider the control of the interconnection of two systems governed by an 1D wave equation, which was considered in [15]. Different from [15], the control inputs $\zeta_{3}(t)$ and $\zeta_{4}(t)$ are given by

$$
\begin{aligned}
\zeta_{3}(t)= & \beta_{1} \xi_{1}(0, t)+\beta_{2} \xi_{2}\left(0, t-\frac{\ell_{2}}{c}\right)+\alpha_{1} \xi_{1}\left(0, t-\frac{\ell_{2}}{c}\right)+\alpha_{2} \xi_{2}(0, t) \\
& +\delta_{1} \xi_{2}\left(0, t-\frac{\ell_{1}-\ell_{2}}{c}\right)+\delta_{2} \xi_{1}\left(0, t-\frac{2 \ell_{1}-\ell_{2}}{c}\right), \\
\zeta_{4}(t)= & \beta_{3} \xi_{1}\left(0, t-\frac{\ell_{1}}{c}\right)+\beta_{4} \xi_{2}(0, t)+\alpha_{3} \xi_{2}\left(0, t-\frac{\ell_{1}}{c}\right)+\alpha_{4} \xi_{1}(0, t) \\
& +\delta_{3} \xi_{1}\left(0, t-\frac{\ell_{2}-\ell_{1}}{c}\right)+\delta_{4} \xi_{2}\left(0, t-\frac{2 \ell_{2}-\ell_{1}}{c}\right) .
\end{aligned}
$$

Then by using the derivation outlined in [15] (see Section 6), the state-space representation $x(t)=\left[\xi_{1}^{\mathrm{T}}(0, t), \xi_{2}^{\mathrm{T}}(0, t)\right]^{\mathrm{T}}$ is governed by

$$
x(t)=A_{1}^{\prime} x\left(t-r_{1}\right)+A_{2}^{\prime} x\left(t-r_{2}\right)+A_{3}^{\prime} x\left(t-r_{3}\right)+A_{4}^{\prime} x\left(t-r_{4}\right),
$$

where $r_{1}=\frac{\ell_{1}}{c}, r_{2}=\frac{2 \ell_{1}}{c}, r_{3}=\frac{\ell_{2}}{c}, r_{4}=\frac{2 \ell_{2}}{c}$, and

$$
\begin{aligned}
& A_{1}^{\prime}=\left[\begin{array}{cc}
-2 \alpha_{4} & 2-2 \beta_{4} \\
0 & -2 \delta_{1}
\end{array}\right], A_{2}^{\prime}=\left[\begin{array}{cc}
-\left(1+2 \beta_{3}\right) & -2 \alpha_{3} \\
-2 \delta_{2} & 0
\end{array}\right], \\
& A_{3}^{\prime}=\left[\begin{array}{cc}
-2 \delta_{3} & 0 \\
2-2 \beta_{1} & -2 \alpha_{2}
\end{array}\right], A_{4}^{\prime}=\left[\begin{array}{cc}
0 & -2 \delta_{4} \\
-2 \alpha_{1} & -\left(1+2 \beta_{2}\right)
\end{array}\right] .
\end{aligned}
$$

Obviously, system (47) is a delay-difference equation in the form of (1) with $N=4$.

Let $\beta_{1}=1.005, \beta_{2}=-0.475, \beta_{3}=-0.6, \beta_{4}=1.15, \alpha_{1}=$ $0.05, \alpha_{2}=-0.025, \alpha_{3}=-0.1, \alpha_{4}=-0.1, \delta_{1}=-0.15, \delta_{2}=$ $0.15, \delta_{3}=-0.025, \delta_{4}=0.05$. Then it can be obtained from (2) that the strong stability radius

$$
\gamma_{1}^{*}:=\sup _{\left(\theta_{1}, \theta_{2}, \theta_{3}, \theta_{4}\right) \in[0,2 \pi]^{4}} \rho\left(\sum_{i=1}^{4} A_{i}^{\prime} \mathrm{e}^{\mathrm{j} \theta_{i}}\right)
$$

of system (47) is 0.6875 . Let $A_{1}=\frac{1}{\gamma_{1}} A_{1}^{\prime}, A_{2}=\frac{1}{\gamma_{1}} A_{2}^{\prime}, A_{3}=$ $\frac{1}{\gamma_{1}} A_{3}^{\prime}, A_{4}=\frac{1}{\gamma_{1}} A_{4}^{\prime}$, where $\gamma_{1} \in \mathbf{R}$ is a free parameter to be determined. Similarly to Example 1, we use Theorem 1, Theorem 1 in [29], Theorem 4 in [14], Theorem 6.1 in [12] to determine the minimum $\gamma_{1}$ such that delay-difference equation (1) is strongly stable. The results are recorded in Table I. It can be seen that $\gamma_{1}$ obtained by Theorem 1 with $k=2$ is smaller than the one obtained by the results in [12], [14] and is the same as the one obtained from (2) and the one obtained by Theorem 1 in [29]. It follows from Table I that Theorem 1 with $k=2$ allows us to obtain much less conservative results than that in [12] and [14].

Table I

THE MINIMUM VALUE OF $\gamma_{1}$ BY USING DIFFERENT METHODS

\begin{tabular}{cc}
\hline Methods & The Minimum Value of $\gamma_{1}$ \\
\hline Theorem 6.1 in [12] & 0.7252 \\
Theorem 4 in [14] & 0.7249 \\
Theorem 1 in [29] & 0.6875 \\
Spectral condition (2) & 0.6875 \\
& 0.7252 (when $k=1)$ \\
Theorem 1 & 0.6875 (when $k=2)$ \\
& 0.6875 (when $k=3)$ \\
\hline
\end{tabular}

Example 3: We consider perturbed delay-difference equation

$$
\begin{aligned}
x(t)= & \left(A_{1}+\Delta A_{1}\right) x\left(t-r_{1}\right)+\left(A_{2}+\Delta A_{2}\right) x\left(t-r_{2}\right) \\
& +\left(A_{3}+\Delta A_{3}\right) x\left(t-r_{3}\right),
\end{aligned}
$$

where $A_{1}, A_{2}, A_{3}$ are given in Example 1 with $\gamma_{0}=1$ and

$$
\begin{aligned}
\Delta A_{1}= & {\left[\begin{array}{llll}
\eta & 0 & 0 & 0 \\
0 & 0 & 0 & 0 \\
0 & 0 & 0 & 0 \\
0 & 0 & 0 & 0
\end{array}\right], \Delta A_{2}=\left[\begin{array}{llll}
0 & 0 & 0 & 0 \\
0 & 0 & \beta & 0 \\
0 & 0 & 0 & 0 \\
0 & 0 & 0 & 0
\end{array}\right], } \\
\Delta A_{3}= & {\left[\begin{array}{llll}
0 & 0 & 0 & 0 \\
0 & 0 & 0 & 0 \\
\vartheta & 0 & 0 & 0 \\
0 & 0 & 0 & 0
\end{array}\right], }
\end{aligned}
$$

where $\eta \in[-\varpi, \varpi], \beta \in[-\varpi, \varpi], \vartheta \in[-\varpi, \varpi]$ with $\varpi>0$ being uncertainties. We choose

$$
\begin{aligned}
& \bar{E}=\left[\begin{array}{c}
I_{3} \\
0_{1 \times 3}
\end{array}\right], \quad \bar{A}_{1}=\left[\begin{array}{cccc}
\varpi & 0 & 0 & 0 \\
0 & 0 & 0 & 0 \\
0 & 0 & 0 & 0
\end{array}\right], \\
& \bar{A}_{2}=\left[\begin{array}{cccc}
0 & 0 & 0 & 0 \\
0 & 0 & \varpi & 0 \\
0 & 0 & 0 & 0
\end{array}\right], \bar{A}_{3}=\left[\begin{array}{cccc}
0 & 0 & 0 & 0 \\
0 & 0 & 0 & 0 \\
\varpi & 0 & 0 & 0
\end{array}\right] \text {, }
\end{aligned}
$$

which correspond to (43) with $F=\operatorname{diag}\left\{\frac{\eta}{\varpi}, \frac{\beta}{\varpi}, \frac{\vartheta}{\varpi}\right\}$. We want to find the maximal value of $\varpi$ (denoted by $\varpi^{*}$ ) such that (41) is strongly stable for all $\eta \in[-\varpi, \varpi], \beta \in[-\varpi, \varpi], \vartheta \in[-\varpi, \varpi]$. 
By using Theorem 3 for different $k$ and by applying a bisection search on $\varpi$, we find that $\varpi^{*}(1)=0.2867$ and $\varpi^{*}(k)=$ $0.2871, k \geq 2$. Thus, the maximal allowable $\varpi$ is found to be 0.2871 by Theorem 3. On the other hand, by using Theorem 2 and by applying once again a bisection search on $\varpi$, we find that the maximal allowable $\varpi$ is 0.4048 , which is larger than 0.2871 . This indicates that there is still room for improvement of our LMIs based sufficient conditions in Theorem 3.

\section{CONCLUSION}

The paper addressed the strong stability problem for delaydifference equations with multiple time-delays. An LMI-based condition was obtained to assess strong stability. A time-domain interpretation of the proposed LMI condition in terms of a quadratic integral Lyapunov functional was given, and the relationship between the proposed condition and an existing result was discussed. A sufficient condition expressed by LMIs and a necessary and sufficient condition, expressed in terms of structured singular values, were presented to guarantee the robust strong stability of the delay-difference equation with norm bounded uncertainties. Numerical examples demonstrated the effectiveness of the established results.

\section{REFERENCES}

[1] P. Appeltans, and W. Michiels, "A pseudo-spectra based characterisation of the robust strong $H_{\infty}$ norm of time-delay systems with real-valued and structured uncertainties," arXiv preprint arXiv:1909.07778, 2019.

[2] C. E. Avellar and J. K. Hale, "On the zeros of exponential polynomials," Journal of Mathematical Analysis and Applications, vol. 73, pp. 434452, 1980.

[3] R. Bellman, K. Cooke, Differential-difference equations. New York, NY, USA: Academic Press, 1963.

[4] P.-A. Bliman, "A convex approach to robust stability for linear systems with uncertain scalar parameters," SIAM Journal on Control and Optimization, vol. 42, no. 6, pp. 2016-2042, 2004.

[5] P.-A. Bliman, "From Lyapunov-Krasovskii functionals for delayindependent stability to LMI conditions for $\mu$-analysis," Advances in Time-Delay Systems, pp. 75-85, 2004.

[6] P.-A. Bliman, "An existence result for polynomial solutions of parameterdependent LMIs," Systems \& Control Letters, vol. 51, pp. 165-169, 2004.

[7] F. Borgioli, W. Michiels, "A novel method to compute the structured distance to instability for combined uncertainties on delays and system matrices," IEEE Transactions on Automatic Control, vol. 65, pp. 17471754, 2019.

[8] R. Brayton, "Nonlinear oscillations in a distributed network," Quarterly of Applied Mathematics, vol. 24, no. 4, pp. 289-301, 1967.

[9] A. Bressan, Hyperbolic systems of conservation laws: the one dimensional cauchy problem. London, U.K.: Oxford Univ. 2000.

[10] V. D. Blondel and A. Megretski, Unsolved problems in mathematical systems and control theory. Press, 2004.

[11] E. R. Campos, S. Mondié, and M. Di Loreto, "Necessary stability conditions for linear difference equations in continuous time," IEEE Transactions on Automatic Control, vol. 63, no. 12, pp. 4405-4412, 2018.

[12] L. A. V. Carvalho, "On quadratic Liapunov functionals for linear difference equations," Linear Algebra and Its Applications, vol. 240, pp. 41-64, 1996.

[13] J.-M. Coron, G. Bastin, and B. d'André-Novel, "Dissipative boundary conditions for one-dimensional nonlinear hyperbolic systems," SIAM Journal on Control and Optimization, vol. 47, no. 3, pp. 1460-1498, 2008.

[14] S. Damak, M. Di Loreto, S. Mondié, and X. Brun, "Exponential stability with decay rate estimation for linear difference equations," IEEE Transactions on Automatic Control, vol. 61, no. 1, pp. 252-257, 2016.

[15] S. Damak, M. Di Loreto, S. Mondié, "Stability of linear continuous-time difference equations with distributed delay: Constructive exponential estimates," International Journal Robust Nonlinear Control, vol. 25, no. 17, pp. 3195-3209, 2015.

[16] S. Damak, M. Di Loreto, W. Lombardi, and V. Andrieu, "Stability analysis for a class of linear systems governed by continuous-time difference equations," Automatica, vol. 50, no. 12, pp. 3299-3303, 2014.
[17] E. Fridman, "New Lyapunov-Krasovskii functionals for stability of linear retarded and neutral type systems," Systems \& Control Letters, vol. 43, pp. 309-319, 2001.

[18] E. Fridman, "Stability of linear descriptor systems with delay: A Lyapunov based approach," Journal of Mathematical Analysis and Applications, vol. 273, pp. 24-44, 2002.

[19] K. Gu, "A review of some subtleties of practical relevance for time delay systems of neutral type," ISRN Applied Mathematics, vol. 46, pp. 253-298, 2012.

[20] K. Gu, "Stability problem of systems with multiple delay channels," Automatica, vol. 46, pp. 743-751, 2010.

[21] J. C. Geromel and R. H. Korogui, "Analysis and synthesis of robust control systems using linear parameter dependent Lyapunov functions," IEEE Transactions on Automatic Control, vol. 51, no. 12, pp. 1984-1989, 2006.

[22] C. Graziano, "Exact robust stability analysis of uncertain systems with a scalar parameter via LMIs," Automatica, vol. 49, no. 4, pp. 1083-1086, 2013.

[23] J. Hale and S. M. Verduyn Lunel, "Strong stabilization of neutral functional differential equations," IMA Journal of Mathematical Control and Information, vol. 19, pp. 5-23, 2002.

[24] J. K. Hale and S. M. Verduyn Lunel, Introduction to functional differential equations. New York, USA: Springer-Verlag, 1993.

[25] D. Henrion and T. Vyhlídal, "Positive trigonometric polynomials for strong stability of difference equations," Automatica, vol. 48, no. 9, pp. 2207-2212, 2012

[26] D. Hinrichsen and A. J. Pritchard, Mathematical Systems Theory I, volume 48 of Texts in Applied Mathematics. Springer Berlin Heidelberg, 2005.

[27] V. De Iuliis, A. Germani, and C. Manes, "Internally positive representations and stability analysis of linear difference systems with multiple delays," IFAC PapersOnLine Volume, vol. 50, no. 1, pp. 3099-3104, 2017.

[28] H. Li and K. Gu, "Discretized lyapunov-krasovskii functional for coupled differential-difference equations with multiple delay channels," Automatica, vol. 46, no. 5, pp. 902-909, 2010.

[29] Z. Li, Q. Fan, L. Li, and Y. Wang, "Stability analysis of linear continoustime delay-difference systems with multiple time-delays," Journal of the Franklin Institute, 2019,10.1016/j.jfranklin.2019.08.004.

[30] Z. Li, J. Lam, and Y. Wang, "Stability analysis of linear stochastic neutral-type time-delay systems with two delays," Automatica, vol. 91, pp. 179-189, 2018.

[31] Q. Li, B. Bernhardsson, A. Rantzer, E. J. Davison, P. M. Young, and J. C. Doyle, "A formula for computation of the real stability radius," Automatica, vol. 31, no. 6, pp. 879-890, 1995.

[32] D. Melchor-Aguilar, "Exponential stability of linear continuous time difference systems with multiple delays," Systems \& Control Letters, vol. 62, pp. 811-818, 2013.

[33] D. Melchor-Aguilar, "On Lyapunov functionals for linear functional difference equations," Systems \& Control Letters, vol. 127, pp. 1-5, 2019.

[34] W. Michiels and T. Vyhlídal, "An eigenvalue based approach for the stabilization of linear time-delay systems of neutral type," Automatica, vol. 41, no. 6, pp. 991-998, 2005.

[35] W. Michiels, T. Vyhlídal, P. Zítek, H. Nijmeijer, and D. Henrion, "Strong stability of neutral equations with an arbitrary delay dependency structure delay dependency structure," SIAM Journal on Control and Optimization, vol. 48, no. 2, pp. 763-786, 2009.

[36] P. Pepe and E. I. Verriest, "On the stability of coupled delay differential and continuous time difference equations," IEEE Transactions on Automatic Control, vol. 48, no. 8, pp. 1422-1427, 2003.

[37] P. Pepe, "On the asymptotic stability of coupled delay differential and continuous time difference equations," Automatica, vol. 41, pp. 107-112, 2005.

[38] A. Rantzer, "On the Kalman-Yakubovich-Popov lemma," Systems \& Control Letters, vol. 28, pp. 7-10, 1996.

[39] V. Rasvan and S. Niculescu, "Oscillations in lossless propagation models: a Liapunov Krasovskii approach," IMA Journal of Mathematical Control and Information, vol. 19, pp. 157-172, 2002

[40] N. Stankovic, S. Olaru, and S.-I. Niculescu, "Further remarks on asymptotic stability and set invariance for linear delay-difference equations," Automatica, vol. 50, no. 8, pp. 2191-2195, 2014.

[41] B. Zhou, "On strong stability and robust strong stability of linear difference equations with two delays," Automatica, vol. 110, 2019.

[42] B. Zhou and Q. Liu, "Input delay compensation for neutral type timedelay systems," Automatica, vol. 78, pp. 309-319, 2017. 